\title{
Uterine neuroendocrine tumors - literature review
}

\author{
Nicolae BACALBASA ${ }^{1,2,3}$, Irina BALESCU ${ }^{2,4}$, Mihaela VILCU ${ }^{2,3}$, Simona DIMA ${ }^{1}$, Iulian BREZEAN ${ }^{2,3}$ \\ ${ }^{1}$ Center of Excellence in Translational Medicine, Fundeni Clinical Institute, Bucharest, Romania \\ 2"Carol Davila" University of Medicine and Pharmacy, Bucharest, Romania \\ 3"Ion Cantacuzino" Clinical Hospital, Bucharest, Romania \\ ${ }^{4}$ Ponderas Academic Hospital, Bucharest, Romania
}

\begin{abstract}
Uterine neuroendocrine tumors are extremely rare malignancies which are usually diagnosed in advanced stages of the disease and are associated with poor prognostic. Due to their rarity a standard therapeutic strategy as well as a standard diagnostic protocol is still missing, these patients being rather diagnosed and treated by extrapolation of the present guidelines for other types of neuroendocrine tumors. This is a literature review of the most relevant studies conducted on the issue of diagnostic and management of uterine neuroendocrine tumors.
\end{abstract}

Keywords: uterine neuroendocrine tumors, surgery, radiotherapy, prognostic

\section{INTRODUCTION}

Primary gynecological neuroendocrine malignancies are rare and aggressive neoplastic diseases which are usually diagnosed in advanced stages of the disease and report a very poor prognostic. It is estimated that less than $2 \%$ of all gynecological malignancies are represented by the neuroendocrine subtype, the most common location being represented by uterine cervix followed by ovary and uterus $(1,2)$. Generally, gynecological neuroendocrine tumors are classified as typical, atypical carcinoids, small cell and large cell neuroendocrine tumors respectively, the histopathological subtype being a significant prognostic factor in regard to the long term outcomes of these patients; therefore, while patients diagnosed with carcinoid tumors report a better outcome, these histopathological subtypes representing in fact well dif- ferentiated malignancies with indolent course, patients presenting small cell or large cell tumors have a significantly poorer outcome due to the biological aggressiveness of this subtype $(1,2)$. Although it seems that in the last decades the incidence of gynecological neuroendocrine tumors has raised, this fact is not entirely true, the increasing incidence being rather related to an improvement of the diagnostic tests (3).

\section{MATERIAL AND METHODS}

Patients diagnosed with uterine neuroendocrine tumors (NET) from 1985 to present were included and were classified according to the International Federation of Obstetrics and Gynecology (FIGO) classification in early stage disease (stages I-II) and advanced stages (stages III- 
IV). Medline research of the English literature between 1982 and 2020 was performed, all articles with the keywords "uterine", "neuroendocrine" "cancer" and "tumor" being reviewed. Further on, all related papers included in their reference list were also reviewed. Due to the extreme rarity of this pathology, case reports and small case series were also included.

\section{HISTOPATHOLOGICAL CLASSIFICATION OF UTERINE NET}

Similarly to other types of NET, uterine NET are classified as low grade carcinoid tumors, high grade small cell NET and large cell NET. Since that moment there have been 20 cases of large cell NET, up to $40 \%$ of them being reported by the Eastern authors $(4,5)$. However, pure large cell NET are rare, most often mixed variants of endometroid, serous adenocarcinoma or carcinosarcoma and large cell NET being encountered (6-9).

\section{DIAGNOSTIC OF UTERINE NET}

Most often patients diagnosed with uterine NET present for diffuse pelvic pain, abnormal uterine bleeding or weight loss especially due to the fact that they are diagnosed in advanced stages of the disease, when distant metastases are already present; therefore is estimated that up to $80 \%$ of cases are diagnosed in advanced stages of the disease while up to half of them are diagnosed when distant metastases are already present (3). Whenever a patient presents abnormal uterine bleeding the first diagnostic step is to perform an endometrial biopsy; however, in cases which are finally diagnosed with uterine NET only an extremely small proportion will be diagnosed after endometrial biopsy (10). As for the utility of serum tumor markers detection, CA125, lactate dehydrogenase and neuron specific enolase have been proposed especially in patients with large cell NET; however these markers failed to demonstrate remarkable sensitivity or specificity in such cases $(10,11)$. In the meantime association of imagistic methods such as pelvic magnetic resonance is not reliable in order to establish the final positive diagnostic due to the fact that most often these patients will present non-specific features such as no delimitation between endometrial lining and myometrium, large uterine masses with areas of necrosis and probably intratumoral hemorrhage (10).

Due to the extremely low possibility of a uterine tumor to be a neuroendocrine one, unfortu- nately a significant number of cases will be initially misdiagnosed and considered as poorly differentiated carcinomas; in order to have a correct and complete diagnostic, in such cases neuroendocrine markers should be tested during the histopathological analysis of the specimen (4). However, it has been stated that the positivity of one neuroendocrine biomarker is enough in order to classify a lesion as a NET; the most commonly suggested such markers are represented by CD56 followed by chromogranin, synaptophysin and neuron specific enolase (10). In the meantime, whenever the suspicion of diagnostic is of an uterine neuroendocrine carcinoma an important step during the diagnostic protocol is To establish if this tumor is a primitive one or is in fact a metastasis originating from other primaries such as from lung cancer. In this respect, when it comes to small cell uterine NET diagnostic criteria for primary uterine tumors include an unequivocal evidence of endometrial origin, dense sheet like growth in standard hematoxylin eosin stain and one or more positive neuroendocrine markers (12).

Interestingly, a significant number of cases will present mixed features of NET and adenocarcinoma of the uterus; however, in such cases it has been suggested that unless the NET component is very reduced the primary classification of these cases should be as NET due to the fact that this component is more likely to influence the long term clinical outcome (13). Once this observation has been stated the number of cases classified as NET significantly increased, leading to the increase of the proportion of such cases.

When it comes to the possible confusions when it comes to the diagnostic of uterine NET, a very interesting situation was described by Ono et al. in 2016; therefor the authors presented the case of a 41-year-old patient with previous history of presumed undifferentiated uterine adenocarcinoma, at that moment a total hysterectomy with bilateral adnexectomy was performed; however two years later she was diagnosed with suspect pulmonary nodules, was submitted to surgery and a upper lobectomy was performed; at that moment the histopathological studies raised the suspicion of large cell NET but the immunohistochemical staining demonstrated the presence of estrogen and progesterone receptors. Moreover, the histopathological characteristics of the uterine and pulmonary tumors were similar; in this respect the final diagnostic was the one of large cell NET combined with areas of endometroid adenocarcinoma and lung metastases originating from the NET component (14). 


\section{MANAGEMENT OF UTERINE NET}

Due to the extremely low number of cases diagnosed with uterine NET a standardized therapeutic protocol is still missing, in certain cases the treatment being in fact extrapolated form small cell pulmonary tumors; therefore the therapeutic strategy proposed for uterine NET include association of surgery, radiotherapy and chemotherapy; however, due to the biological aggressiveness of these lesions most cases are diagnosed in advanced stages of the disease and have a very poor prognostic $(3,15)$. In the meantime, extrapolating therapeutic strategies from other types of NET leaded to a preliminary conclusion that the most efficient therapeutic strategy might consist of surgery followed by radiotherapy. However, it seems that up to $70 \%$ of patients diagnosed with uterine NET are submitted to surgery as initial therapeutic strategy (3).

In cases diagnosed with small cell neuroendocrine tumors of the uterine body most often the first intent therapeutic option consists of surgery followed by adjuvant chemotherapy and radiotherapy; however the overall prognostic remains very poor, most cases diagnosed so far presenting recurrent disease within the first two years of treatment (9,16-19). However, the biological aggressiveness of these lesions explains the high propensity to spread rapidly through the lymphatic and hematogenous pathways leading to the apparition of large, disseminated adenopathic masses as well as of parenchimatous metastases at the level of the liver, lungs and even brain (20).

When it comes to the utility of adjuvant chemotherapy, it seems that the most efficient therapeutic strategy proposed so far consists of a combination of platinum salts such as cisplatin and etoposide or irinotecan (21).
Due to the improvement of the diagnostic and therapeutic strategies, it has been demonstrated that the mean survival interval has increased in the last decades, from 42.9 months for patients diagnosed before the year of 2004 and 47.7 months for patients diagnosed after this moment $(p=0.44)(3)$. When it comes to large cell NET, a recent study came to underline the fact that at three year after the initial diagnostic none of the cases having this diagnostic was not disease free, the longest disease free survival reported so far being of 20 months (4).

\section{CONCLUSIONS}

Uterine NET represent a very rare and aggressive biological subtype of uterine malignancies which are usually diagnosed in advanced stages of the disease; due to the extremely low number of cases reported so far standard therapeutic strategy is still missing; however it seems that extrapolation of the protocol from other types of NET might provide an improvement of the overall survival. Therefore, most cases reported so far have been treated by cytoreductive surgery followed by adjuvant radiotherapy as a stand-alone procedure or in association with chemotherapy. However, prospective studies are still needed in order to establish the most appropriate therapeutic strategy and to investigate the potential role of targeted therapies.

\section{Acknowledgement}

This work was supported by the project entitled "Multidisciplinary Consortium for Supporting the Research Skills in Diagnosing, Treating and Identifying Predictive Factors of Malignant Gynecologic Disorders", project number PN-IIIP1-1.2-PCCDI2017-0833.

\section{REFERENCES}

1. Rouzbahman M, Clarke B. Neuroendocrine tumors of the gynecologic tract: Select topics. Semin.Diagn.Pathol. 2013; 30:224-233.

2. Patibandla JR, Fehniger JE, Levine DA, Jelinic P. Small cell cancers of the female genital tract: Molecular and clinical aspects. Gynecol.Oncol. 2018; 149:420-427.

3. Gibbs J, Mei S, Economos K, Lee YC et al. Clinicopathologic features, incidence, and survival trends of gynecologic neuroendocrine tumors: A SEER database analysis. Am.J.Obstet.Gynecol. 2019; 221:53.

4. Jenny C, Kimball K, Kilgore L, Boone J. Large cell neuroendocrine carcinoma of the endometrium: A report and review of the literature. Gynecol.Oncol.Rep. 2019; 28:96-100.

5. Matsumoto H, Nasu K, Kai K et al. Combined large-cell neuroendocrine carcinoma and endometrioid adenocarcinoma of the endometrium: $A$ case report and survey of related literature. J.Obstet.Gynaecol.Res. 2016; 42:206-210.

6. Tamura T, Jobo T, Watanabe J, Kanai T et al. Neuroendocrine features in poorly differentiated endometrioid adenocarcinomas of the endometrium. Int.J.Gynecol.Cancer 2006; 16:821-826.

7. Mulvany NJ, Allen DG. Combined large cell neuroendocrine and endometrioid carcinoma of the endometrium. Int.J.Gynecol.Pathol. 2008; 27:49-57.

8. Posligua L, Malpica A, Liu J et al. Combined large cell neuroendocrine carcinoma and papillary serous carcinoma of the endometrium with pagetoid spread. Arch. Pathol.Lab Med. 2008; 132:1821-1824.

9. Froio E, D'Adda T, Fellegara $\mathrm{G}$ et al. Uterine carcinosarcoma metastatic to the lung as large-cell neuroendocrine carcinoma with synchronous sarcoid granulomatosis. Lung Cancer 2009; 64:371-377.

10. Hiroshima K, Mino-Kenudson M. Update on large cell neuroendocrine carcinoma. Transl.Lung Cancer Res. 2017; 6:530-539. 
11. Isgro M A, Bottoni P, Scatena R. NeuronSpecific Enolase as a Biomarker: Biochemical and Clinical Aspects. Adv.Exp. Med.Biol. 2015; 867:125-143.

12. van Hoeven $\mathrm{KH}$, Hudock JA, Woodruff JM, Suhrland MJ. Small cell neuroendocrine carcinoma of the endometrium. Int.J.Gynecol.Pathol. 1995; 14:21-29.

13. Albores-Saavedra J, Gersell D, Gilks CB et al. Terminology of endocrine tumors of the uterine cervix: Results of a workshop sponsored by the College of American Pathologists and the National Cancer Institute. Arch.Pathol.Lab Med. 1997; 121:34-39.

14. Ono K, Yokota N R, Yoshioka E et al. Metastatic large cell neuroendocrine carcinoma of the lung arising from the uterus: A pitfall in lung cancer diagnosis. Pathol.Res.Pract. 2016; 212:654-657.
15. Reed NS, Gomez-Garcia E, GallardoRincon D et al. Gynecologic Cancer InterGroup (GCIG) consensus review for carcinoid tumors of the ovary. Int.J.Gynecol. Cancer 2014; 24:S35-S41.

16. Manivel C, Wick MR, Sibley RK. Neuroendocrine differentiation in mullerian neoplasms. An immunohistochemical study of a "pure" endometrial small-cell carcinoma and a mixed mullerian tumor containing small-cell carcinoma. Am.J.Clin.Pathol. 1986; 86:438-443.

17. Olson N, Twiggs L, Sibley R. Small-cell carcinoma of the endometrium: Light microscopic and ultrastructural study of a case. Cancer 1982; 50:760-765.

18. Katahira A, Akahira J, Niikura H, Ito K, Moriya T, Matsuzawa S, Makinoda S, Oda T, Fujiwara K, Yaegashi N. Small cell carcinoma of the endometrium: Report of three cases and literature review. Int.J.Gynecol.Cancer 2004; 14:1018-1023.

19. Huntsman DG, Clement PB, Gilks CB, Scully RE. Small-cell carcinoma of the endometrium. A clinicopathological study of sixteen cases. Am.J.Surg.Pathol. 1994; 18:364-375.

20. D'Antonio A, Addesso M, Caleo A, Guida M, Zeppa P. Small cell neuroendocrine carcinoma of the endometrium with pulmonary metastasis: A clinicopathologic study of a case and a brief review of the literature. Ann.Med.Surg.(Lond) 2016; 5: 114-117.

21. Zivanovic O, Leitao MM Jr, Park KJ et al. Small cell neuroendocrine carcinoma of the cervix: Analysis of outcome, recurrence pattern and the impact of platinum-based combination chemotherapy. Gynecol.Oncol. 2009; 112:590-593.

\section{Conflict of interest: none declared} Financial support: none declared 